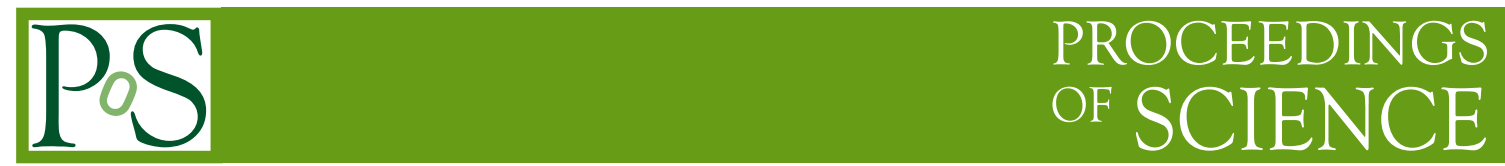

\title{
A different view on light element anti-correlations in globular clusters: fluorine abundances in NGC 6656 (M22)
}

Valentina D'Orazi*

Macquarie University

Monash Centre for Astrophysics

E-mail: valentina.dorazi@mq.edu.au

Maria Lugaro, George Angelou

Monash Centre for Astrophysics

Sara Lucatello, Raffaele G. Gratton

INAF Osservatorio di Padova

Angela Bragaglia, Eugenio Carretta

INAF Osservatorio di Bologna

Alan Alves-Brito

Australian National University

Inese I. Ivans

The University of Utah

Thomas Masseron

Universitè libre de Brussels

Alessio Mucciarelli

Università di Bologna 
Observed chemical (anti)correlations in the light elements $\mathrm{C}, \mathrm{N}, \mathrm{O}, \mathrm{Na}, \mathrm{Mg}$, and $\mathrm{Al}$ among globular cluster (GC) stars are presently recognised as the signature of self-pollution from previous generations of stars. This defines the multiple population scenario (MPS). Since fluorine is involved in the complete $\mathrm{CNO}$ cycle, determining its abundance in GCs provides new and complementary clues regarding the nature of these previous generations. In fact, along with the $\mathrm{Na}-\mathrm{O}$ and $\mathrm{Mg}-\mathrm{Al}$ anti-correlations, the MPS also predicts an anti-correlation between $\mathrm{F}$ and $\mathrm{Na}$ and a positive correlation between $\mathrm{F}$ and $\mathrm{O}$. Furthermore, theoretical models suggest that low-mass $\left(\lesssim 5 \mathrm{M}_{\odot}\right)$ asymptotic giant branch (AGB) stars are $\mathrm{F}$ producers (and are also responsible for $\mathrm{C}+\mathrm{N}+\mathrm{O}$ and $s$-process element variations). We present our results on near-infrared CRIRES spectroscopic observations of six giant stars in the metal-poor GC NGC 6656 (M22): we aim at inferring the F content and its (possible) variation in this $\mathrm{GC}$, which exhibits a rather peculiar abundance pattern, with variations in both light $(\mathrm{C}, \mathrm{N}, \mathrm{O}, \mathrm{Na}, \mathrm{Mg}, \mathrm{Al})$ and heavy (Fe-peak and $s$-process) elements.

XII International Symposium on Nuclei in the Cosmos

August 5-12, 2012

Cairns, Australia

* Speaker. 


\section{Scientific framework}

A large variety of studies have revealed the complex nature of elemental abundances in GCs. Abundance variations in the lighter elements ( $\mathrm{Li}, \mathrm{C}, \mathrm{N}, \mathrm{O}, \mathrm{Na}, \mathrm{Mg}$ and $\mathrm{Al}$ ), recognised since the 1970's (e.g., [1]), demand that some material must have been processed through the entire hot CNO cycle: element pairs $\mathrm{C}-\mathrm{N}, \mathrm{O}-\mathrm{Na}$, and $\mathrm{Mg}-\mathrm{Al}$ are anti-correlated such that the abundances of $\mathrm{C}, \mathrm{O}$ and $\mathrm{Mg}$ are depleted and $\mathrm{N}, \mathrm{Na}$ and $\mathrm{Al}$ are enhanced. It is now well accepted that this pattern is related to a self-enrichment mechanism due to a previous generation of stars, whereby those elements affected by proton-captures and ultimately responsible for the observed anti-correlations, are synthesised in the stellar interiors. The origin of the polluters responsible is still debated (intermediatemass AGB stars by [12] or fast rotating massive stars by [2]). The global picture is further complicated by the presence of some peculiar clusters, the most famous case being $\omega$ Centauri (see e.g., [3]), where variations in Fe-peak and s-process elements have also been observed. The metalpoor GC M22 $([\mathrm{Fe} / \mathrm{H}]=-1.70)$ is one such GC. Analysing 35 giant stars, Marino and collaborators ([7]) found that this GC is comprised of two distinct stellar groups, characterised by an offset in metallicity and in $s$-process element content, namely $\Delta[\mathrm{Fe} / \mathrm{H}]=0.15 \pm 0.02$ and $\Delta[s / \mathrm{Fe}]=0.36 \pm 0.02$ dex. These authors suggested that the weak s-process component, activated in massive stars $(\mathrm{M} \gtrsim$ $25 \mathrm{M}_{\odot}$ ) during core He-burning and C-shell phases, may have played a role in the observed abundance patterns. This is in contrast to a recent study by [9] who focused on the heavy element content (from $\mathrm{Y}$ to $\mathrm{Th}$ ) across the two stellar sub-groups and ruled out the massive star origin. We decided to approach the problem from a different perspective, deriving fluorine $(\mathrm{F})$ abundances for a sample of six cool giant stars in M22. These stars were carefully selected from both sub-stellar groups as defined by [7]. The F content is a powerful tracer of the polluter mass range in M22 because its production/destruction is highly dependent on the stellar mass. Theoretical models of AGB stars predict that $\mathrm{F}$ is produced from the reaction ${ }^{18} \mathrm{O}(\mathrm{p}, \alpha){ }^{15} \mathrm{~N}(\alpha, \mathrm{p}){ }^{19} \mathrm{~F}$ in the He intershell during thermal pulses associated with He burning. The peak of F production in AGB stars is reached for stars of initial masses $\sim 2 \mathrm{M}_{\odot}$ [5]. In AGB stars with masses larger than roughly $5 \mathrm{M}_{\odot}$, and depending on the metallicity, $\mathrm{F}$ is destroyed both via $\alpha$ captures in the He intershell, and via proton captures at the base of the convective envelope due to hot bottom burning (HBB). Moreover, AGBs undergoing $\mathrm{HBB}$ can also destroy $\mathrm{O}$ and $\mathrm{Mg}$ and produce $\mathrm{Na}$ and $\mathrm{Al}$. Thus, according to the MPS, we should expect the abundances of $\mathrm{F}$ to be correlated with $\mathrm{O}$ (and $\mathrm{Mg}$ ) and anti-correlated with those of $\mathrm{Na}$ (and Al). This prediction was indeed observationally confirmed in the GCs M4 and NGC 6712 by [11] and [13].

\section{Sample, observations, and abundance analysis}

Our sample includes six Red Giant Branch (RGB) stars (stellar parameters, Na, O, and La abundances were determined by [7]): three stars belong to the metal-poor (MP, s-process poor) population and three stars to the metal-rich (MR, s-process rich) one, as according to [7]. We employed the high-resolution near-IR spectrograph CRIRES@VLT, with a 0.4" slit $(\mathrm{R}=50,000)$, covering a wavelength range of 22950-23520 , which includes the unblended HF feature at $23358 \AA$, as well as several CO lines and the Na I line at 23379 A. For each target, the spectrum of an early-type star was acquired, aimed to perform the telluric feature subtraction. The abundance analysis was 


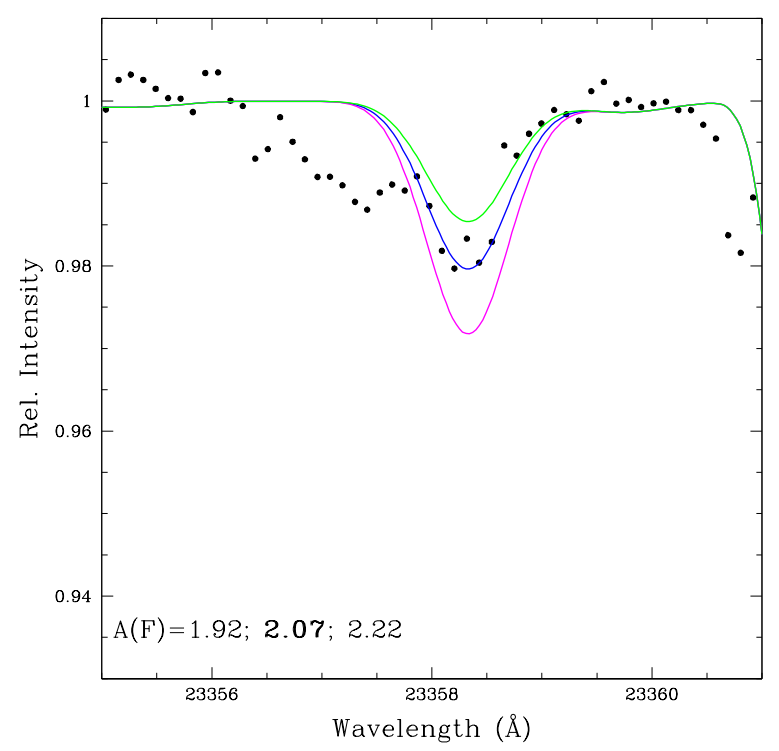

Figure 1: Spectral synthesis for the HF line for one of our sample stars.

carried out using the driver synth in MOOG ([10]) and the Kurucz's set of model atmospheres ([4], with no overshooting. An example of the spectral synthesis for the HF feature is given in Fig. 1. Internal errors affecting our $\mathrm{F}$ abundances are mainly due to the adopted set of stellar parameters and to the best-fit determination. The sensitivity of the $\mathrm{F}$ abundance to input stellar parameters was evaluated by separately changing effective temperature, surface gravity and microturbulence values; we obtained a typical uncertainty of $0.11 \mathrm{dex}$ in $[\mathrm{F} / \mathrm{H}]$. Errors due to the best-fit determination (owed mainly to the $\mathrm{S} / \mathrm{N}$ of the spectra) are instead $\pm 0.07 \mathrm{dex}$. The total internal error is then calculated by summing in quadrature both uncertainties $(0.13 \mathrm{dex})$.

\section{Results and discussion}

We detected star-to-star variations in $\mathrm{F}$ abundances, with values ranging from $[\mathrm{F} / \mathrm{H}]=-2.82$ dex to $[\mathrm{F} / \mathrm{H}]=-2.23$ (i.e., a factor of $\sim 4$ ). Such a large spread is beyond that of the measurement uncertainties (see previous Section). Moreover, the variations are not random but are positively correlated with $\mathrm{O}$ and anti-correlated with $\mathrm{Na}$, as shown in Fig. 2. The observed chemical pattern can be explained by the presence of H-burning (via the $\mathrm{CNO}$ cycle) at high temperatures which leads to the destruction of $\mathrm{F}$ in conjunction with $\mathrm{O}$ depletion and $\mathrm{Na}$ enhancement. Interestingly, those (anti)correlations are detectable in each of the M22 sub-components (the $s$-rich and s-poor groups). 

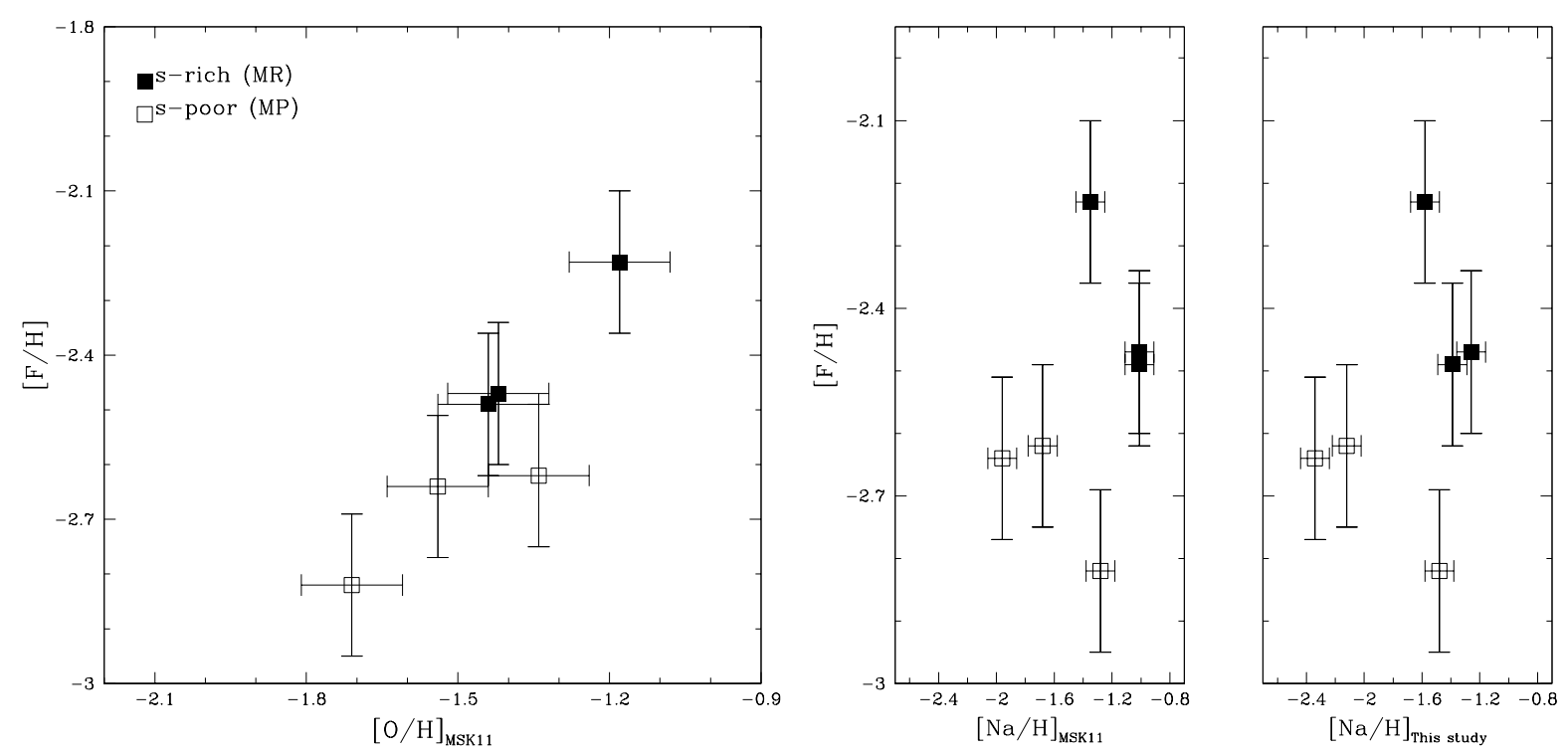

Figure 2: Run of $\mathrm{F}$ with $\mathrm{O}$ (left-hand panel) and $\mathrm{Na}$ (right-hand panel; we show both our Na values from infra-red spectroscopy and the optical ones derived by [7]). Metal-poor (s-poor) and metal-rich (s-rich) stars are labelled as empty and filled symbols, respectively.

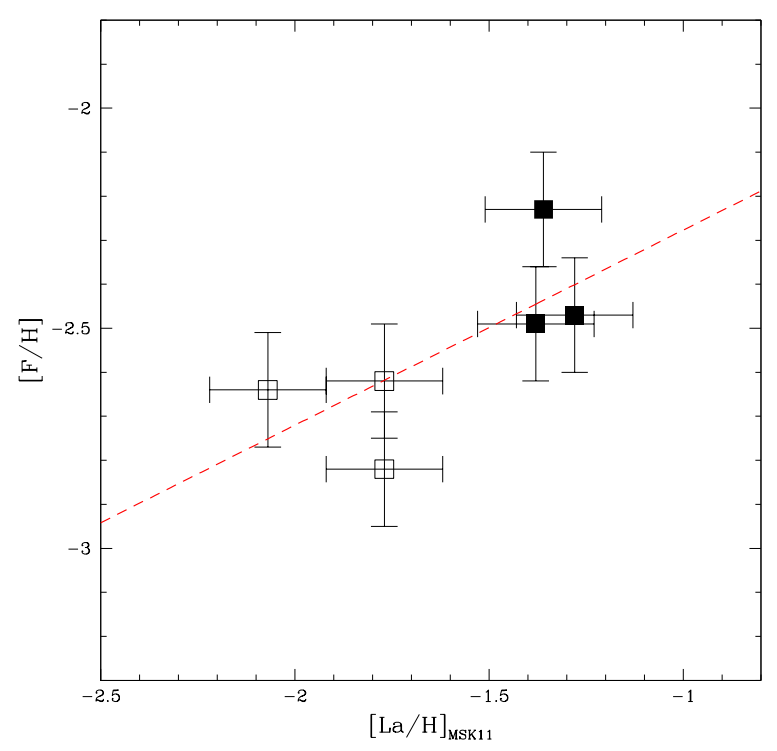

Figure 3: $[\mathrm{F} / \mathrm{H}]$ as a function of $[\mathrm{La} / \mathrm{H}]$ for our sample of six stars. 
Beyond the internal spread in F characterising each sub-component, we measured an increase in the F content between the two different stellar generations in M22. The s-process-rich group has, on average, larger $F$ abundances than the s-process-poor group. This is shown in Fig. 3, where we plot our $\mathrm{F}$ abundances $([\mathrm{F} / \mathrm{H}])$ as a function of $[\mathrm{La} / \mathrm{H}]$ from [7]. There is a positive correlation between the two ratios, suggesting that the polluters responsible for the $s$-process production must also account for the $\mathrm{F}$ production.

Comparing our abundances with models by [6] we found that AGB stars with masses of $\approx$ 4-5 $\mathrm{M}_{\odot}$ can reproduce the observed pattern. Interestingly, [9] reached the same conclusion by exploring the heavy-element ratios, such as $[\mathrm{Pb} / h s]^{1}$. Thus, observational constraints from both light (F) and heavy elements point to the same polluter mass range between the different stellar groups in M22, (i.e. AGBs with masses around $4-5 \mathrm{M}_{\odot}$ ), and imply a difference in age no larger than a few hundred Myr. Notably, Marino and colleagues chemically characterised the double sub-giant branch (SGB) of this cluster, and concluded that the age spread can be at most $\sim 300$ Myr.

\section{References}

[1] Cohen 1978, ApJ, 223, 487

[2] Decressin, T., Meynet, G., Charbonnel, C., Prantzos, N., Ekström, S. 2007, A\&A, 464, 1029

[3] Johnson, C.I., Pilachowski, C.A. 2010, ApJ, 722, 1373

[4] Kurucz, R. 1993, CD-ROM 13, ATLAS9 Stellar Atmosphere Programs and 2 km/s Grid (Cambridge: $\mathrm{SAO}), 13$

[5] Lugaro, M., Ugalde, C., Karakas, A.I., et al. 2004, ApJ, 615, 934

[6] Lugaro, M., Karakas, A.I., Stancliffe, R.J., Rijs, C. 2012, ApJ, 747, 2

[7] Marino, A. F., Sneden, C., Kraft, R.P., et al. 2011, A\&A, 532, A8 (MSK11)

[8] Marino, A.F., Milone, A., Sneden, C., et al. 2012, A\&A, 541, 15

[9] Roederer, I.U., Marino, A.F., Sneden, C. 2011, ApJ, 742, 37

[10] Sneden, C. 1973, ApJ, 184, 839

[11] Smith, V.V., Cunha, K., Ivans, I.I., et al. 2005, ApJ, 633, 392

[12] Ventura, P., D’Antona, F., Mazzitelli, I., Gratton, R.G. 2001, ApJ, 550, L65

[13] Yong, D., \& Grundahl, F. 2008, ApJ, 672, L29

\footnotetext{
${ }^{1} h s$ is for second-peak $s$-process elements, e.g., Ba, La, Ce.
} 